\title{
Danger of delay for genetic tests
}

\section{Molecular biology is providing novel means of testing for genetic disease, with the result that researchers are having to spend their time on repetitive testing. Now is the time to make better plans.}

THE detractors of molecular biology used to complain that its discoveries might be interesting in an academic fashion, but would have no clinical significance. But recent years have shown that position to be untenable. Indeed, the growing impact of molecular biology on medicine is nurturing a band of clinicians with a deep interest in research while bringing molecular biologists face to face with some of the realities of disease. So much was clear, for example, at the latest International Titisee Conference (24-27 April) on human gene mapping and molecular pathology; progress is rapid, in both the laboratory and the clinic. But it will need a great deal of care, at least in Europe, if present enthusiasms are not to dissipate under the weight of that age-old resentment in which clinicians unthinkingly demand more (and different) diagnostic testing from researchers who believe, rightly or otherwise, that they have better ways in which to spend their time.

The high crest of the common ground between the laboratory and the clinic is undoubtedly, just now, the hunt for the unidentified genes whose defects are responsible for diseases such as cystic fibrosis and muscular dystrophy for which no molecular basis has as yet been determined. The magnitude of this task should not be underestimated, but, as Peter Little explains on page 558, adequate techniques are now available, if not perfected. Each gene has been located in a rather small fragment of a chromosome, but which corresponds to a stretch of DNA which is long by the yardstick of what can now be sequenced. Yet there should not be too long to wait before the first of them is identified.

The molecular probes used in location of these genes are already valuable in the prenatal diagnosis of carriers of defective genes, for the time being only within families in which the disease is known to be present. So long as the genes themselves have not been identified, success depends on a variety of factors - how many independent gene probes there are, how close they lie to the defective gene and whether there are enough close relatives of the two parents of the fetus who are 'informative' in the genetic sense. For some diseases, there is clearly room for improvement, but for many others diagnosis is already reliable. So there is a growing demand, by physicians on behalf of their patients, for tests to be carried out. Already the demand sometimes outstrips the time and people available, not to mention the people's interest in work that may seem routine.

The procedures are far from simple. Obtaining the prenatal samples is often the least of the problems, but may sometimes be limiting. This may be especially so as pressure grows to replace sampling of the amniotic fluid by sampling of the placenta as a means of obtaining fetal material, which in principle allows diagnosis earlier in pregnancy and thus earlier termination (if indicated). Curiously, although chorionic villus sampling of the placenta was originally thought to be more risky (for the fetus) than amniocentesis, experience has tended to reverse that view, although chorionic villus sampling is still relatively uncommon.

Sampling, however done, is for physicians. It is the samples that demand the immediate attention and the experience of the researchers. For the time being, most of them are likely to be eager to devote time and energy to this task, not only for humanitarian reasons but also, where the still-cryptic genetic diseases are concerned, because of the possibility that any new case may turn out to have a particular genetic defect that provides a short cut to the defective gene itself.

For each disease, this captive enthusiasm of the researchers must be a passing phase. The time must come when they want to move on to other problems. When, for example, the defective gene for cystic fibrosis or muscular dystrophy is identified, the laboratory emphasis is certain to shift to the function of the protein encoded by the gene and to the links between that function and the symptoms of the disease. Because these links are likely to lead both to new discoveries in fundamental science and to new approaches to therapy, it is in everybody's interest that they should be investigated. But who will then take over the presumably increased diagnostic load?

Even if prenatal diagnosis is confined to the industrialized countries, the imagination is challenged by the sheer scale on which testing may be required if the potential of the new discoveries is to be as fully exploited as preventive medicine. Tests which are at present complicated will no doubt be simplified as time goes on, but by then there will be other defective genes for which to test.

The problems occasioned by diseases that run in families may be relatively small, requiring as they do the testing only of family (including embryonic family) members, most probably at specialist centres which they attend already, so that there is a persuasive argument that the costs of diagnosis and even of the requested termination that may follow will be more than offset by what is saved in there being fewer afflicted patients to treat. But nobody will be surprised if this argument runs into the hidden buffers that so often hinder the application of preventative medicine.

How much greater will be the problems when it becomes feasible and seems to be desirable to screen whole populations for diseases that most often arise as new mutations, not as inherited genetic defects? Or what happens when the several genetic factors contributing to susceptibility to cardiovascular disease have been identified, a question now at the focus of a great deal of research? It should then be possible to categorize groups of people who are at risk, and who could (and should) take preventive steps, but only by complicated population screening.

At the rate at which this science is progressing, the diagnostic needs occasioned even by the simple single-gene diseases are almost certain to outstrip the creation of new testing facilities within public health-care systems, nowhere expansionist. Will medical charities feel compelled to spend less on research and more on testing? Commercial services will certainly grow to meet the need; in the United States, biotechnology companies seem bent on exploiting an emerging market, while, even in Britain, ICI's investment in a centre for providing DNA fingerprinting tests seems well set for moving to meet the growing demand of sophisticated prenatal diagnostic tests.

But where will that leave those who cannot afford the going commercial cost of tests? Especially when the need, in many industrialized communities, is most evident among immigrant populations? It will be frustrating for those laboratory scientists who have helped to open up this new vein of preventative medicine if they are faced, a few years from now, with the unpalatable choice of devoting themselves to routine analysis of a kind that they alone are able to perform or, alternatively, to see the potential benefits of their research simply run to waste.

Peter Newmark 